\title{
Activation of Divergent Neuronal Cell Death Pathways in Different Target Cell Populations during Neuroadapted Sindbis Virus Infection of Mice
}

\author{
MICHAEL B. HAVERT, ${ }^{1}$ BRIAN SCHOFIELD, ${ }^{2}$ DIANE E. GRIFFIN, ${ }^{1,3}$ AND DAVID N. IRANI ${ }^{1,3 *}$ \\ W. Harry Feinstone Department of Molecular Microbiology and Immunology ${ }^{1}$ and Department of Environmental \\ Health Science, ${ }^{2}$ Johns Hopkins University School of Hygiene and Public Health, Baltimore, Maryland 21205, and \\ Department of Neurology, Johns Hopkins University School of Medicine, Baltimore, Maryland $21287^{3}$
}

Received 6 October 1999/Accepted 10 March 2000

\begin{abstract}
Infection of adult mice with neuroadapted Sindbis virus (NSV) results in a severe encephalomyelitis accompanied by prominent hindlimb paralysis. We find that the onset of paralysis parallels morphologic changes in motor neuron cell bodies in the lumbar spinal cord and in motor neuron axons in ventral nerve roots, many of which are eventually lost over time. However, unlike NSV-induced neuronal cell death found in the brain of infected animals, the loss of motor neurons does not appear to be apoptotic, as judged by morphologic and biochemical criteria. This may be explained in part by the lack of detectable caspase-3 expression in these cells.
\end{abstract}

Sindbis virus (SV) is an alphavirus, a member of the togavirus family. It is transmitted naturally to a variety of hosts by insect vectors and was originally isolated in 1952 from mosquitoes in Egypt (21). In the laboratory, SV causes an acute encephalomyelitis when inoculated into mice, leading to prominent neuronal infection. A strain of SV more neurovirulent for mice was developed by serial passage of the original isolate in mouse brain (6). Following intracerebral inoculation, this neuroadapted strain (NSV) causes a severe, often fatal, encephalomyelitis accompanied by prominent hindlimb paralysis in adult mice $(7,8)$. Paralysis develops as a result of infection that spreads to motor neurons of the lumbosacral spinal cord which innervate the hindlimb musculature (7). The pathogenesis of this hindlimb paralysis is not completely understood; while some motor neuron degeneration has been observed in NSV-infected mice (8), other animals have been reported to recover neurologic function following infection $(4,7,13)$. Our present study was carried out to determine whether paralysis results from the loss of function or the actual degeneration of infected lumbar motor neurons.

We find that NSV-induced paralysis typically begins in C57BL/6 mice inoculated with $10^{3}$ PFU of virus after 4 days of infection, and all mice show some degree of paralysis by day 5 . Paralysis is initially mild with decreased hindlimb movement, but as the disease worsens, all animals become severely paralyzed and unable to move their hindlimbs in response to a pain stimulus. After 10 days, more than $90 \%$ of infected mice had died.

To determine how lumbar motor neurons in NSV-infected mice were affected in comparison to the kinetics with which hindlimb paralysis developed, we examined $2-\mu \mathrm{m}$-thick plasticembedded sections of spinal cord by light microscopy. The cell bodies of motor neurons in uninfected mice exhibited a characteristic morphology, with large nuclei, dispersed chromatin, and prominent nucleoli and Nissl substance (Fig. 1A). At day

\footnotetext{
* Corresponding author. Mailing address: Department of Molecular Microbiology and Immunology, The Johns Hopkins University School of Hygiene and Public Health, 615 N. Wolfe St., Baltimore, MD 21205-2179. Phone: (410) 955-3726. Fax: (410) 955-0105. E-mail: dirani@jhmi.edu.
}

3 of infection, most motor neurons had an overall morphology that was similar to that of uninfected cells. However, subtle pathologic changes, including mild swelling with an increase in cytoplasmic vacuolation, were noted in some of these cells (Fig. 1B). These changes progressed to severe abnormalities over several days and correlated with the onset and increasing severity of hindlimb paralysis. Six days after infection, many motor neurons had a swollen, ghost-like appearance (Fig. 1C). Both the cytoplasm and the nuclei of these cells were enlarged and had lost most of their normal staining characteristics. The nuclear membrane in severely damaged cells had lost its integrity, making it difficult to identify as a discrete structure (Fig. 1C). By 9 days after infection, the cytoplasmic membrane had dissolved, leaving a hole in the tissue section (Fig. 1D). A large number of these holes could be observed in the ventral horn of the spinal cord at this stage. These observations show that motor neurons in the lumbar spinal cord are lost during the course of NSV infection and that the morphology is not consistent with classical apoptosis.

To further study this process of motor neuron loss, we examined ventral nerve roots of the spinal cord. Because each of these nerve roots is comprised exclusively of the axons which originate from motor neurons at adjacent levels in the lumbar spinal cord, we reasoned that this would allow us to rapidly survey the fate of a large number of these cells. The nerve roots in question run parallel to the spinal cord before exiting the spinal canal and could be easily visualized in sections made through the entire spinal column (Fig. 2A). Ventral nerve roots were identified based on their position relative to the spinal cord and their uniform axonal morphology. To visualize the axons in each nerve root, silver staining was performed by a modified Bielschowsky method (23). Individual ventral nerve roots from the L4-L5 level of uninfected mice (Fig. 2B) and mice 3, 6, 9, and 28 days after infection with NSV (Fig. $2 \mathrm{C}$ to F) were examined at higher magnification. No evidence of axonal damage was apparent in the ventral roots of uninfected animals or in animals 3 days after NSV infection (Fig. 2B and C). Axonal damage first became evident 6 days after infection, coincident with the progression of hindlimb paralysis and the appearance of swollen cell bodies within the ventral horn of the spinal cord; many of these axons appeared engorged with 

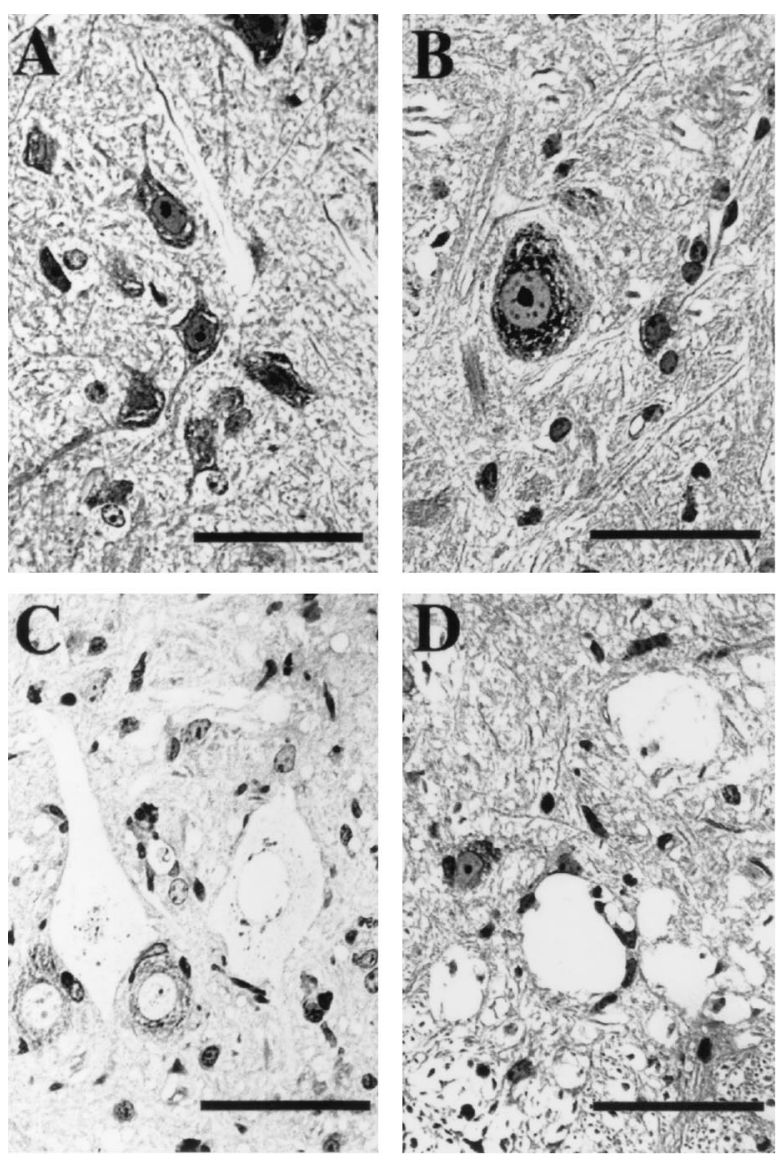

FIG. 1. Morphologic features of motor neurons in the lumbar spinal cord of NSV-infected mice. Two-micrometer-thick sections of spinal cord from the L4-L5 vertebral level were prepared from an uninfected animal (A) and animals 3 (B), 6 (C), and 9 (D) days after infection. Bars $=50 \mu \mathrm{m}$. Tissue sections were stained with toluidine blue. Uninfected motor neurons appeared histologically normal (A), but 3 days after infection, some cells began to show subtle pathologic changes, including mild swelling and cytoplasmic vacuolization (B). By day 6 , many motor neurons were severely swollen and more lightly stained (C), and by day 9 , the nuclear and cytoplasmic membranes had dissolved, leaving numerous empty holes in the spinal cord tissue (D). vacuoles (Fig. 2D). Axons in the dorsal roots did not show these changes, indicating that sensory neurons are unaffected by NSV infection (data not shown). Axonal swelling was more severe in lumbar ventral roots after 9 days (Fig. 2E), but an actual loss of axons was difficult to judge at this and earlier time points. After 28 days in the few paralyzed animals which had survived the acute infection, swollen axons were no longer present, and significant axonal loss in the lumbar ventral roots was seen (Fig. 2F). We conclude that axonal degeneration occurs in a delayed manner and over a longer interval than the loss of motor neuron cell bodies, but that motor neurons and their processes are eliminated following infection with NSV.

SV-induced neuronal cell death has previously been described to be apoptotic in nature as judged by morphologic, genetic, and biochemical criteria (reviewed in reference 5). Motor neurons have not been specifically investigated in these studies, and the morphologic evidence presented in Fig. 1 suggests that neuronal loss in the spinal cord does not occur through a typical apoptotic process. To pursue this possibility, we analyzed spinal cord sections by using a terminal transferase-mediated nick-end labeling (TUNEL) technique to detect fragmented DNA in combination with viral immunohistochemistry to identify infected cells. After 3 days of infection, many virus-positive cells were detected (Fig. 3A). Virus antigen was found predominantly in ventral horn cells with motor neuron morphology. While a few TUNEL-positive cells were observed at this time, TUNEL signal was not detected in infected neurons (Fig. 3A). In the lumbar spinal cords of animals 6 days after NSV infection, abundant TUNEL signal and virus antigen expression were both detected (Fig. 3B). While some TUNEL staining was found in proximity to virus antigen, a considerable amount was also present in areas relatively devoid of infection. As a result, we suggest that many cells other than infected neurons are dying by a mechanism that involves DNA fragmentation. In areas of heavy virus antigen deposition, we were unable to definitively identify the cellular source of the TUNEL signal, because infected neuronal cell bodies were no longer apparent.

To better understand the source of the TUNEL signal in the spinal cord, double-labeling experiments using other cell-type-
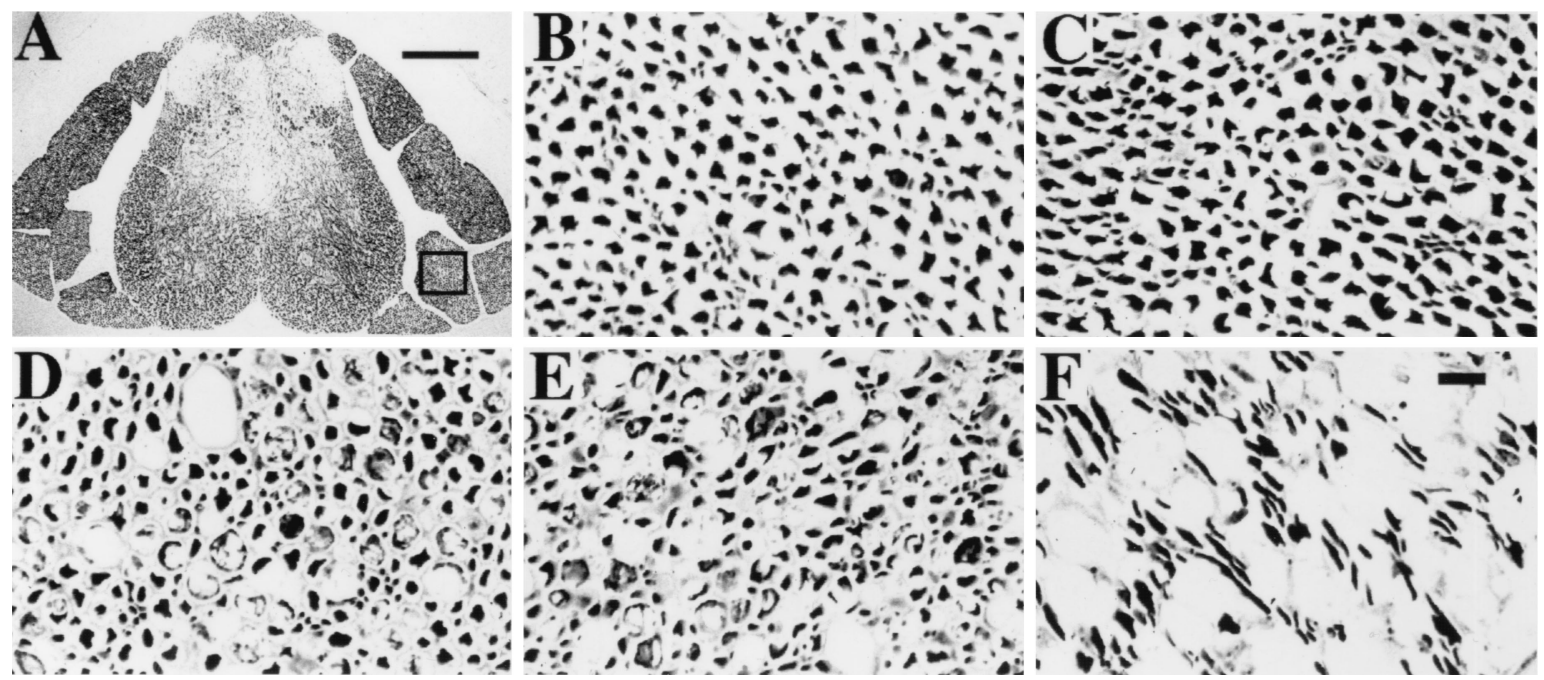

FIG. 2. Appearance of motor neuron axons in ventral nerve roots of NSV-infected mice. Spinal cord sections of the L4-L5 vertebrae were collected after infection with NSV, and axons were identified by silver staining. (A) Cross-section of the entire spinal column of an uninfected mouse. An area of a ventral nerve root is present within the box. Bar $=200 \mu \mathrm{m}$. (B to F) Axons in individual ventral roots from an uninfected mouse (B) and from animals 3 (C), 6 (D), 9 (E), and 28 (F) days after infection. Bar $=10 \mu \mathrm{m}$. 


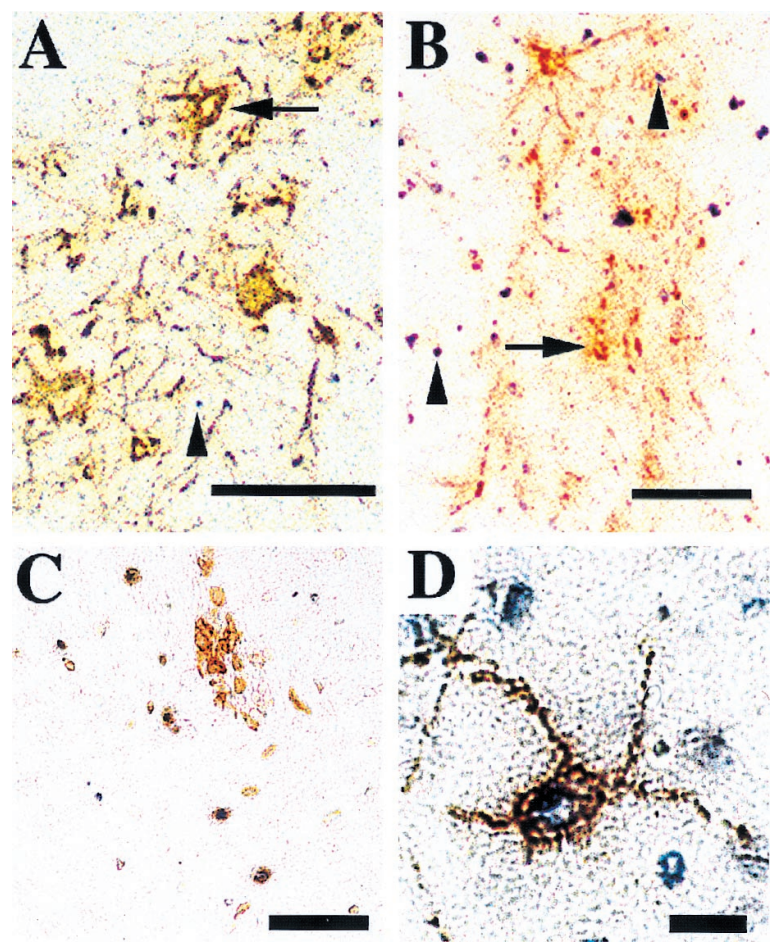

FIG. 3. Staining of lumbar spinal cord sections during NSV infection by combined TUNEL-viral antigen immunohistochemistry. (A) The ventral horn of the lumbar spinal cord 3 days after NSV infection. Virus antigen (brown) is present in neuronal cell bodies (arrow) and axons. TUNEL signal (blue) can be seen in cells around areas of virus infection (arrowhead). Bar $=100 \mu \mathrm{m}$. (B) The ventral horn of the lumbar spinal cord 6 days after NSV infection. Virus antigen appears clumped (arrow), and discrete cell bodies are poorly seen. Abundant TUNEL signal is present both within and outside areas of virus antigen (arrowheads). Bar $=100 \mu \mathrm{m}$. Combined TUNEL and immunohistochemistry using a cocktail of B- and T-cell specific antibodies [CD45R/B220, CD8a(ly-2), and CD4(L3T4)] reveals a cluster of lymphocytes (brown), presumably around a blood vessel in the spinal cord (C). TUNEL signal is present within some of these cells. Bar $=50 \mu \mathrm{m}$. (D) TUNEL signal present in an infected neuron of the spinal cord 3 days postinfection that had been treated with DNase I. Bar $=20$ $\mu \mathrm{m}$.

specific markers were performed. We identified infiltrating lymphocytes as one source of this TUNEL signal (Fig. 3C) and believe that apoptosis of recruited inflammatory cells accounts for much of the TUNEL staining in the spinal cord on day 6 of infection. Because DNA fragmentation may happen at different stages of apoptosis (1), this process could have occurred in infected neurons sometime between day 3 and day 6 of infection. Multiple attempts to colocalize TUNEL signal within infected neurons on days 4 and 5 of infection were also unsuccessful (data not shown). To rule out the possibility that the lack of detectable TUNEL signal in infected neurons was the result of a technical problem with our assay, spinal cord sections were treated with DNase before labeling. Under these conditions, TUNEL signal was easily detected in virus-infected neurons, suggesting that colocalization can be achieved by our double-labeling technique (Fig. 3D). From these results, we suggest that infected motor neurons are degenerating in the absence of significant DNA fragmentation.

One explanation for why motor neurons do not appear to undergo apoptosis during NSV infection is that these particular cells do not express the intracellular machinery required for this type of cell death. Because caspase-3 plays a critical role in directly activating an endogenous endonuclease during apoptosis $(3,14)$, we performed tissue immunohistochemistry with an antibody specific for the cleaved (activated) form of caspase-3 (19). Activated caspase-3 should only be detected in cells undergoing apoptosis, and we found that staining was not detected in spinal cord sections from uninfected mice (data not shown). Significant levels of activated caspase-3 were also not detected in motor neurons on either day 4 or day 6 of NSV infection when the loss of motor neurons occurs (Fig. 4A and B). Consistent with our observed pattern of TUNEL staining, activated caspase- 3 was detected in cells that appeared to be infiltrating lymphocytes (Fig. 4B). When staining was performed with another antibody that detects both the uncleaved and cleaved forms of caspase-3, expression in motor neurons from uninfected mice was largely undetectable, consistent with previous reports (10). Staining was easily detected, however, in cells with an astrocytic morphology present mostly in spinal cord white matter regions (Fig. 4C). Caspase-3 expression was also not induced above background in motor neurons after infection (Fig. 4D). Signal could, however, be detected in cells that appeared to be infiltrating lymphocytes and glial cells (Fig. 4D).

Because previous studies have shown that virus-induced neuronal apoptosis occurs in the brains of SV-infected mice (11-13), we analyzed brain tissue sections from NSV-infected animals to validate the techniques used in our study. Neurons

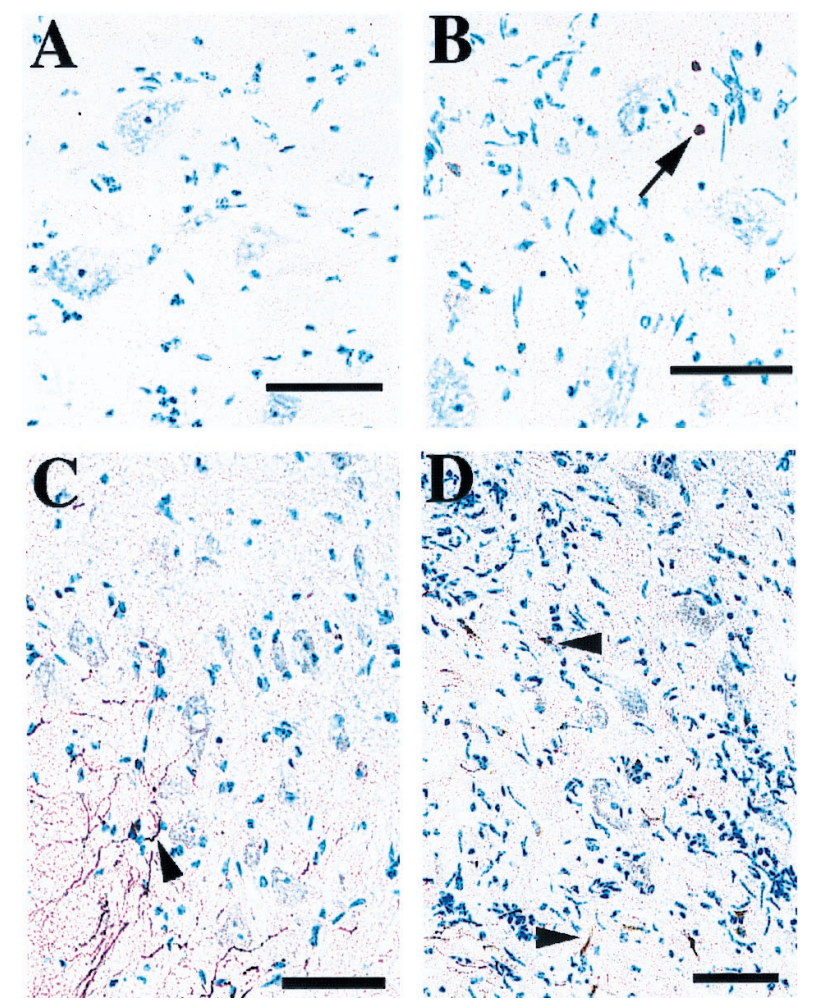

FIG. 4. Caspase-3 immunohistochemistry in the lumbar spinal cord of NSVinfected mice. Frozen sections of lumbar spinal cord were counterstained with methylene blue, identifying large motor neuron cell bodies (light blue) in the ventral horn. Bar $=50 \mu \mathrm{m}$. Activated caspase- 3 was not detected with CM1 immune serum (Idun Pharmaceuticals, Inc., La Jolla, Calif.) in spinal cord sections 4 days after NSV infection (A). Six days after infection, activated caspase-3 (brown) was detected in a few cells with a lymphocytic morphology (arrow), but not in motor neurons (B). In uninfected animals, pro-caspase-3 (brown) was detected in white matter regions (arrowhead), but was largely undetectable in motor neurons by a polyclonal caspase- 3 antiserum (Pharmingen, San Diego, Calif.) (C). Six days after infection, pro-caspase-3 (brown) still was not clearly detected in motor neurons, but remained visible in other cell types (arrowheads) (D) 

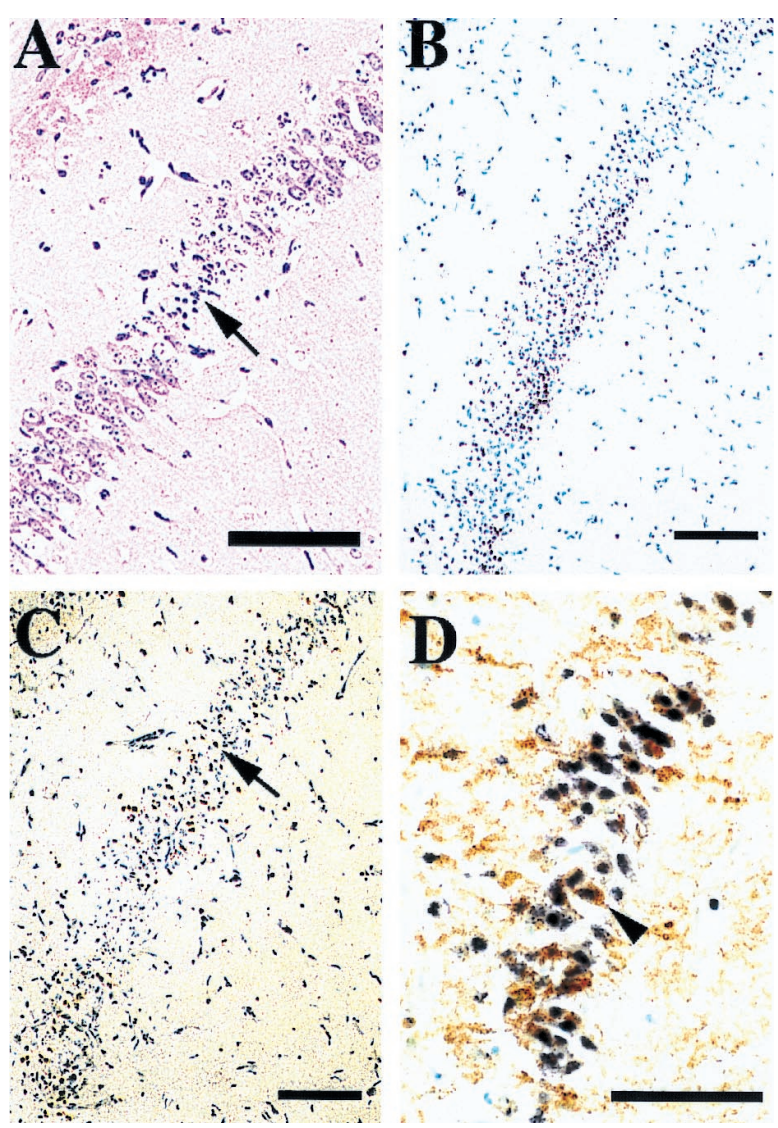

FIG. 5. Analysis of hippocampal regions from NSV-infected mice. (A) Semithin plastic section of mouse brain stained with toluidine blue and naphthol yellow. Bar $=200 \mu \mathrm{m}$. Condensed apoptotic bodies are apparent within the hippocampus (arrow). (B) Abundant TUNEL signal (brown) is also present in the hippocampus of an animal 6 days after infection. Bar $=200 \mu \mathrm{m}$. (C) Activated caspase-3 (brown) is expressed within the same brain region. Bar = $200 \mu \mathrm{m}$. (D) Combined TUNEL-SV immunohistochemistry shows double labeling in neurons (arrowhead) within the hippocampus. Bar $=50 \mu \mathrm{m}$.

in the hippocampus become heavily infected with SV $(8,11$, $13)$, and in plastic sections of this brain region, apoptoticappearing neurons could be found (Fig. 5A). Many of these hippocampal neurons also showed abundant TUNEL staining (Fig. 5B), and the cells frequently expressed the activated form of caspase-3 (Fig. 5C). Colocalization studies showed that many virus antigen-positive neurons in this region were also TUNEL positive (Fig. 5D). As a result, we conclude that the same process which causes motor neuron cell loss in the spinal cord through a nonapoptotic mechanism also induces classical neuronal apoptosis in the brain.

Why different death pathways are activated in motor neurons and some hippocampal neurons during NSV infection warrants further investigation. We suggest, however, that biochemical differences between these two cell types may extend to their endogenous cell death machinery. More specifically, there may be regional variability in the brain regarding the expression of intracellular proteins that mediate virus-induced apoptosis. Consistent with this hypothesis, we were unable to detect activated caspase-3 expression in degenerating motor neurons at the same time that it could be found in apoptotic hippocampal neurons. This difference may be analogous to other experimental systems in which a given stimulus that is normally apoptotic induces nonapoptotic cell death in the ab- sence of caspase activity or the presence of caspase inhibitors $(2,15,22)$. This is supported by our localization studies with caspase-3, an enzyme that is essential for cell death involving DNA fragmentation $(9,20)$.

The results reported here are also consistent with other paradigms of neuronal cell death in which the underlying mechanism can vary according to both the type and state of maturation of the cell being studied. Thus, certain populations of neurons in experimental animals exposed to the same neurotoxic stimulus appear to undergo necrosis, while others appear to be dying by classical apoptosis $(18,24)$. This may, in part, result from different stages of differentiation among the target populations. Perhaps because of the importance of apoptosis in normal nervous system development, immature neurons generally are much more susceptible to apoptosis than mature neurons $(16,17,24)$. Based on the results reported here, caution is urged in assuming that different populations of neurons die by the same mechanism when exposed to the same death stimulus.

We thank Takashi Kimura for helpful suggestions and discussions and Judith Coram for plastic tissue sectioning. We also thank Anu Srinivasan (Idun Pharmaceuticals, Inc.) for generously providing the activated caspase-3 immune serum.

This work was supported by grants from the Muscular Dystrophy Association (D.E.G.) and National Institutes of Health (NS18596) (D.E.G.). M.B.H. is the recipient of an NIH postdoctoral fellowship (NS10924).

\section{REFERENCES}

1. Allen, R. T., W. J. Hunter, and D. K. Agrawal. 1997. Morphological and biochemical characterization and analysis of apoptosis. J. Pharmacol. Toxicol. Methods 37:215-228

2. Amarante-Mendes, G. P., D. M. Finucane, S. J. Martin, T. G. Cotter, G. S. Salvesen, and D. R. Green. 1998. Anti-apoptotic oncogenes prevent caspasedependent and independent commitment for cell death. Cell Death Differ. 5:298-306.

3. Enari, M., E. Sakahira, H. Yokoyama, K. Okawa, A. Iwamatsu, and S. Nagata. 1998. A caspase-activated DNase that degrades DNA during apoptosis, and its inhibitor ICAD. Nature 391:43-50.

4. Gorrell, M. D., J. A. Lemm, C. M. Rice, and D. E. Griffin. 1997. Immunization with nonstructural proteins promotes functional recovery of alphavirusinfected neurons. J. Virol. 71:3415-3419.

5. Griffin, D. E., and J. M. Hardwick. 1997. Regulators of apoptosis on the road to persistent alphavirus-infected neurons. Annu. Rev. Microbiol. 51:565-592.

6. Griffin, D. E., and R. T. Johnson. 1977. Role of the immune response in recovery from Sindbis virus encephalitis in mice. J. Immunol. 118:1070-1075.

7. Jackson, A. C., T. R. Moench, D. E. Griffin, and R. T. Johnson. 1987. The pathogenesis of spinal cord involvement in the encephalomyelitis of mice caused by neuroadapted Sindbis virus infection. Lab. Investig. 56:418-423.

8. Jackson, A. C., T. R. Moench, B. D. Trapp, and D. E. Griffin. 1988. Basis of neurovirulence in Sindbis virus encephalomyelitis of mice. Lab. Investig. 58:503-509.

9. Janicke, R. U., M. L. Sprengart, M. R. Wati, and A. G. Porter. 1998. Caspase-3 is required for DNA fragmentation and morphological changes associated with apoptosis. J. Biol. Chem. 273:9357-9360.

10. Krajewska, M., H.-G. Wang, S. Krajewski, J. M. Zapata, A. Shabaik, R. Gascoyne, and J. C. Reed. 1997. Immunohistochemical analysis of in vivo patterns of expression of CPP32 (caspase-3), a cell death protease. Cancer Res. 57:1605-1613.

11. Lewis, J., G. A. Oyler, K. Ueno, Y. R. Fannjiang, B. N. Chau, J. Vornov, S. J. Korsmeyer, S. Zou, and J. M. Hardwick. 1999. Inhibition of virus-induced neuronal apoptosis by Bax. Nat. Med. 5:832-835.

12. Lewis, J., S. L. Wesselingh, D. E. Griffin, and J. M. Hardwick. 1996. Alphavirus-induced apoptosis in mouse brains correlates with neurovirulence. J. Virol. 70:1828-1835.

13. Liang, X. H., J. E. Goldman, H. H. Jiang, and B. Levine. 1999. Resistance of interleukin-1 $\beta$-deficient mice to fatal Sindbis virus encephalitis. J. Virol. 73:2563-2567.

14. Liu, X., H. Zou, C. Slaughter, and X. Wang. 1997. DFF, a heterodimeric protein that functions downstream of caspase-3 to trigger DNA fragmentation during apoptosis. Cell 89:175-184.

15. McCarthy, N. J., M. K. B. Whyte, C. S. Gilbert, and G. I. Evan. 1997. Inhibition of Ced/ICE-related proteases does not prevent cell death induced by oncogenes, DNA damage, or Bcl-2 homologue Bak. J. Cell Biol. 136:215227. 
16. Portera-Cailliau, C., D. L. Price, and L. J. Martin. 1997. Non-NMDA and NMDA receptor-mediated excitotoxic neuronal deaths in adult brain are morphologically distinct: further evidence for an apoptosis-necrosis continuum. J. Comp. Neurol. 378:88-104.

17. Sammin, D. J., D. Butler, G. J. Atkins, and B. J. Sheahan. 1999. Cell death mechanisms in the olfactory bulb of rats infected intranasally with Semliki Forest virus. Neuropathol. Appl. Neurobiol. 25:236-243.

18. Sloviter, R. S., E. Dean, A. L. Sollas, and J. H. Goodman. 1996. Apoptosis and necrosis induced in different hippocampal neuron populations by repetitive perforant path stimulation in the rat. J. Comp. Neurol. 366:516-533.

19. Srinivasan, A., K. A. Roth, R. O. Sayers, K. S. Schindler, A. M. Wong, L. C. Fritz, and K. J. Tomaselli. 1998. In situ immunodetection of activated caspase-3 in apoptotic neurons in the developing nervous system. Cell Death Differ. 5:1004-1016.

20. Tang, D., and V. J. Kidd. 1998. Cleavage of DFF-45/ICAD by multiple caspases is essential for its function during apoptosis. J. Biol. Chem. 273: 28549-28552.

21. Taylor, R. M., H. S. Hurlbut, T. H. Work, J. R. Kingston, and T. E. Frothingham. 1955. Sindbis virus: a newly recognized arthropod-transmitted virus. Am. J. Trop. Med. Hyg. 4:844-862.

22. Vercammen, D., R. Beyaert, G. Denecker, V. Goossens, G. Van Loo, W. Declercq, J. Grooten, W. Fiers, and P. Vandenabeele. 1998. Inhibition of caspases increases the sensitivity of L929 cells to necrosis mediated by tumor necrosis factor. J. Exp. Med. 187:1477-1485.

23. Yamamoto, T., and A. Hirano. 1986. A comparative study of modified Bielschowsky, Bodian and thioflavin S stains on Alzheimer's neurofibrillary tangles. Neuropathol. Appl. Neurobiol. 12:3-9.

24. Yue, X., H. Mehnet, J. Penrice, C. Cooper, E. Cady, J. S. Wyatt, E. O. R. Reynolds, E. A. Edwards, and M. V. Squier. 1997. Apoptosis and necrosis in the newborn piglet brain following transient cerebral hypoxia-ischaemia. Neuropathol. Appl. Neurobiol. 23:16-25. 